\title{
Smart Projectile State Estimation Using Evidence Theory
}

\author{
Jonathan Rogers* \\ Texas A\&M University, College Station, Texas 77843 \\ and \\ Mark Costello \\ Georgia Institute of Technology, Atlanta, Georgia 30332 \\ DOI: $10.2514 / 1.55652$
}

\begin{abstract}
Smart projectile state estimation is a challenging task due to highly nonlinear vehicle dynamic behavior and unreliable or noisy sensor feedback. Although Kalman-filter-based algorithms are currently the primary means of sensor fusion and state estimation for smart weapons applications, they are limited in estimation accuracy, their ability to combine data from a wide variety of sensors, and their ability to recognize and reject erroneous feedback. This paper explores the use of evidence theory (or Dempster-Shafer theory) for projectile state estimation purposes. Evidence theory offers a generalized framework capable of incorporating feedback from a wide variety of sensors with little a priori knowledge about sensor noise characteristics. The framework's main strengths are its ability to manage varying levels of dynamic uncertainty between sensors and its capability to recognize and report conflicting sensor feedback. The paper begins with an overview of evidence theory and a discussion of its strengths when applied to projectile state estimation. Then an evidence-theory-based filter is described, and an example roll-angle estimator is presented. Results are discussed and performance is compared with an extended Kalman filter. The ability to identify and eliminate malfunctioning sensors from the estimation process proves to be a key advantage of the proposed design over current methods.
\end{abstract}

\begin{tabular}{|c|c|c|}
\hline & & \\
\hline$b$ & $=$ & magnetometer bias \\
\hline $\mathbf{I}_{B}, \mathbf{J}_{B}, \mathbf{K}_{B}$ & $=$ & $\begin{array}{l}\text { unit vectors in the projectile body-fixed frame } \\
B\end{array}$ \\
\hline $\mathbf{I}_{\mathrm{NR}}, \mathbf{J}_{\mathrm{NR}}, \mathbf{K}_{\mathrm{NR}}$ & $=$ & unit vectors in the projectile no-roll frame NR \\
\hline & $=$ & unit vectors in the sensor reference frame $S$ \\
\hline$K_{R}$ & $=$ & roughening coefficient \\
\hline$k_{R}$ & $=$ & $\begin{array}{l}\text { tuning parameter for particle roughening } \\
\text { procedure }\end{array}$ \\
\hline$k_{\varnothing}$ & $=$ & belief assigned to empty set \\
\hline$M_{N}, M_{E}, M_{D}$ & $=$ & $\begin{array}{l}\text { north, east, down components of Earth's } \\
\text { magnetic field }\end{array}$ \\
\hline & $=$ & maximum distance between particles \\
\hline$\tilde{M}_{x}, \tilde{M}_{y}, \tilde{M}_{z}$ & $=$ & $\begin{array}{l}\text { Earth's magnetic field components resolved in } \\
\text { body frame }\end{array}$ \\
\hline$M_{J}^{*}, M_{K}^{*}$ & $=$ & $\begin{array}{l}\mathbf{J}_{B} \text {-aligned and } \mathbf{K}_{B} \text {-aligned single-axis } \\
\text { magnetometer outputs }\end{array}$ \\
\hline $\bar{M}_{J}^{*}, \bar{M}_{K}^{*}$ & $=$ & normalized magnetometer outputs \\
\hline$N_{S}, N_{i}, N_{\Theta}$ & $=$ & $\begin{array}{l}\text { number of particles resampled from singleton, } \\
\text { nonsingleton, and uncertainty belief } \\
\text { assignments }\end{array}$ \\
\hline$n_{\text {mag }}$ & $=$ & magnetometer Gaussian noise \\
\hline$S$ & $=$ & magnetometer scale factor \\
\hline $\mathbf{s}$ & $=$ & distance vector from solar sensor to sun \\
\hline $\bar{v}^{*}$ & $=$ & normalized thermopile sensor output \\
\hline$x_{k, i}^{-}, x_{k, i}^{+}$ & $=$ & $\begin{array}{l}\text { particle before and after resampling at time } \\
\text { step } k\end{array}$ \\
\hline $\begin{array}{l}\gamma_{i j}, \gamma_{i k} \\
\Theta\end{array}$ & $\begin{array}{l}= \\
=\end{array}$ & $\begin{array}{l}\text { half-angles defining solar sensor field of view } \\
\text { frame of discernment }\end{array}$ \\
\hline
\end{tabular}

Presented at the AIAA Atmospheric Flight Mechanics Conference, Portland, OR, 8-11 August 2011; received 17 July 2011; revision received 20 September 2011; accepted for publication 21 September 2011. Copyright $\odot$ 2011 by the American Institute of Aeronautics and Astronautics, Inc. All rights reserved. Copies of this paper may be made for personal or internal use, on condition that the copier pay the $\$ 10.00$ per-copy fee to the Copyright Clearance Center, Inc., 222 Rosewood Drive, Danvers, MA 01923; include the code 0731-5090/12 and $\$ 10.00$ in correspondence with the CCC.

*Assistant Professor, Department of Aerospace Engineering. Member AIAA.

†Sikorsky Associate Professor, School of Aerospace Engineering. Member AIAA.

$$
\begin{array}{ll}
\phi, \theta, \psi & = \\
\phi_{M} & \text { projectile Euler roll, pitch, and yaw angles } \\
& \text { phase angle relating magnetic field and body } \\
\text { frame }
\end{array}
$$

\section{Introduction}

TCREASING availability of low-cost robust sensor technology has enabled weapons designers to outfit gun-launched projectiles with full guidance and control capability. However, due to size, cost, and performance constraints, smart weapons systems typically cannot leverage precise Global Positioning System (GPS) and inertial measurement unit technology developed for missiles and aircraft. Instead, weapons designers have turned to component-level integration of low-cost microelectromechanical systems (MEMS) sensors such as accelerometers and rate sensors, magnetometers, thermopiles, and other small robust sensors for an alternative state estimation solution. Although this sensor integration approach offers significant cost and size advantages, data fusion algorithms are challenged to combine inputs from sensors with widely varying signal characteristics, update rates, and measurement confidence. These difficulties, combined with highly nonlinear vehicle dynamics caused by high spin rates or discontinuous actuation techniques, have limited estimation accuracy achievable by standard extended Kalman filters. There is a critical need to develop new nonlinear filtering techniques that can combine incomplete feedback from a wide variety of sensors, handle dynamic sensor reconfiguration, and produce accurate state estimates in the presence of large measurement uncertainty, unreliable sensors, and nonlinear vehicle dynamics.

For the most part, the challenge of designing the next generation of state estimation algorithms lies in combining nonlinear dynamic models with advanced information fusion techniques that eschew Gaussian error assumptions. Recently, many researchers have been pursuing alternative nonprobabilistic information fusion methods for use in nonlinear estimators (for instance, Dempster-Shafer theory [1-3], possibility theory [4,5], and fuzzy set theory [6,7]). Although belief combination and decision-making differ across each of these methods, they collectively form an alternative to traditional Bayesian filtering in their rigorous treatment of uncertainty and ability to assign evidential weight to nontraditional feedback information. Recently, a framework called finite set statistics (FISST) has been proposed [8-10] that attempts to unify many nonprobabilistic 
methods under a single framework similar to standard Bayesian statistics. The advantage of such a unifying theory is that FISST can be applied to multisensor, multitarget fusion problems using wellknown probabilistic formalisms without knowledge of the rather complex theory of random set mathematics. The so-called probability hypothesis density filter [11] is an implementation of FISST that has been used successfully in multisensor fusion and multitarget tracking problems.

This paper explores the use of a relatively simple nonprobabilistic technique called evidence theory [1-3] (or, equivalently, DempsterShafer theory). Evidence theory is a generalized Bayesian framework that offers a technique to choose between a set of propositions given evidence provided from a variety of sources. It is unique in that it allows assignment of support not only to single propositions, but also to finite sets of propositions. A key strength of evidence theory is that no restrictions are placed on evidential data-information need not even be numerical. Although the framework enables an algebraic manipulation of quantities of evidence, the link between these quantities and the original evidential data, whether numerical or otherwise, is left completely general and is determined by the estimator designer. Thus, sensor fusion can be performed with unprecedented flexibility since the framework can incorporate any piece of data useful to the overall estimation problem.

Over the past several decades, numerous researchers have investigated the use of evidence theory for a wide variety of sensor fusion applications, including geoscience image analysis [12], autonomous mobile robots $[13,14]$, context-sensing humancomputer interaction [15,16], and engine fault diagnosis [17]. Most recently, Zribi et al. [18] introduced an evidence-theory-based architecture for vehicle localization, effectively combining GPS, odometer, and digital map data to determine an estimate for twodimensional vehicle location. An underlying theme throughout this previous research is that data is often combined from a diverse set of sensors offering incomplete information at varying levels of accuracy.

This paper develops an evidence-theory-based state estimator for smart projectile applications. Several key elements of the proposed design prove advantageous compared with traditional smart weapons estimation methods. First, the estimator's use of evidence theory mitigates the need to provide highly accurate a priori sensor error distributions, which can be difficult to obtain for many nontraditional sensor components included in MEMS constellations. In addition, sensor conflict is characterized and used within the estimator to identify and remove malfunctioning sensors from the estimation process. Finally, the estimator uses evidence theory to combine belief assigned not only to single states but also to indiscriminate groups of states, allowing it to rigorously handle nontraditional information like heuristic knowledge of the vehicle trajectory or very-lowresolution sensor feedback. The paper begins with a brief overview of evidence theory and how it has been applied to the sensor fusion problem. Then an evidence-theory-based estimator is developed given an arbitrary number of sensors. Resampling methods and sensor conflict measures are described in detail. An example roll estimator is presented, and estimation results for an example trajectory are compared with those from an extended Kalman filter. This example demonstrates the ability of the evidence-theory-based estimator to combine a wide variety of sensor inputs and handle dynamic reconfiguration when various sensors are deemed unreliable or inoperative.

\section{Overview of Evidence Theory}

\section{A. Theoretical Background}

The mathematical theory of evidence, initially developed by Dempster [1,2], presents a framework for combining sources of information to generate a joint inference based on all available pieces of data. A key strength of this nonprobabilistic approach is that it can rigorously incorporate information other than that provided from digitized sensor feedback: for instance, heuristic knowledge about the likely vehicle trajectory or discrete sensors that provide bounds on possible states but lack the resolution to provide highly accurate information. Furthermore, evidence theory provides the means to assign belief to an uncertainty class that provides useful information regarding estimation error.

In the Dempster-Shafer reasoning system, all possibilities for a given outcome (or state estimate in this case) are enumerated in a socalled frame of discernment. For instance, consider a case in which a state estimator wishes to determine vehicle position in a single dimension among three possibilities given by $x_{1}, x_{2}$, or $x_{3}$. The frame of discernment considers the possibility of each state individually as well as any possible combination of states and is given by

$\Theta$

$$
=\left\{\left\{x_{1}\right\},\left\{x_{2}\right\},\left\{x_{3}\right\},\left\{x_{1} \text { or } x_{2}\right\},\left\{x_{2} \text { or } x_{3}\right\},\left\{x_{1} \text { or } x_{3}\right\},\left\{x_{1} \text { or } x_{2} \text { or } x_{3}\right\}, \varnothing\right\}
$$

where $\varnothing$ denotes the possibility that the location is something other than $x_{1}, x_{2}$, or $x_{3}$ (for the sake of notational simplicity, the set brackets around singleton sets $x_{1}, x_{2}$, and $x_{3}$ will be dropped for the remainder of the paper). Note that the frame of discernment covers all possibilities for the state and thus contains a total of $2^{N}$ possibilities where $N$ is the number of singleton propositions. Each sensor contributes its information by distributing its belief over $\Theta$. This assignment by sensor $S_{i}$ represents the sensor's belief in a given mutually exclusive proposition called the belief mass function and is represented by $m_{i}$. According to sensor $S_{i}$, the overall probability that the vehicle is located at $x_{1}$ lies somewhere in the interval

$$
\operatorname{prob}_{i}\left(x_{1}\right)=\left[\operatorname{bel}_{i}\left(x_{1}\right), \operatorname{pl}_{i}\left(x_{1}\right)\right]
$$

where bel $_{i}\left(x_{1}\right)$ represents the belief confidence and $\mathrm{pl}_{i}\left(x_{1}\right)$ represents the plausibility confidence. The belief confidence accounts for all evidence from sensor $S_{i}$ that directly supports the proposition $x_{1}$ and is given by the summation of belief masses that point to proposition $x_{1}$,

$$
\operatorname{bel}_{i}\left(x_{1}\right)=\sum_{A \subseteq x_{1}} m_{i}(A)
$$

where $A$ in this case represents a focal element of $\Theta$. The plausibility function, which describes the degree of support for all propositions that include $x_{1}$, is given by

$$
\operatorname{pl}_{i}\left(x_{1}\right)=1-\operatorname{bel}_{i}\left(\bar{x}_{1}\right)=\sum_{A \cap x_{1} \neq \varnothing} m(A)
$$

where $\bar{x}_{1}$ represents the complement of set $x_{1}$. The definitions of belief and plausibility can be applied equally to singleton and nonsingleton sets.

Although sensors and sources of information can assign belief to both single states and groups of states (singleton and nonsingleton propositions in the parlance of evidence theory), they can also assign belief to an uncertainty class represented by the frame of discernment itself, $\Theta$. This represents the reluctance to provide any information at all about the state (for instance, if a GPS receiver cannot obtain a navigation solution, it would be incapable of offering useful position information). Since a sensor's belief assignment must total unity, a sensor that could not currently offer any information about the state would assign zero belief to all elements of the frame of discernment and a belief of 1 to the uncertainty class $\Theta$.

Evidence theory provides a method for combining sensor $S_{i}$ observation $m_{i}$ with sensor $S_{j}$ observation $m_{j}$ called Dempster's rule of combination (also called the conjunctive combination rule):

$$
\left(m_{i} \oplus m_{j}\right)\left(x_{1}\right)=\frac{\sum_{A \cap B=x_{1}} m_{i}(A) m_{j}(B)}{1-\sum_{A \cap B=\varnothing} m_{i}(A) m_{j}(B)}
$$

Using this rule, belief functions between two sensors can be combined, and the joint belief distribution is normalized such that $\left(m_{i} \oplus m_{j}\right)(\varnothing)$ is 0 . Because the conjunctive rule is both commutative and associative [19], it can be used iteratively to combine sensor 
inputs from an arbitrary number of sources. Note that determination of the belief mass value $m_{i}$ from sensor information is left completely general and can be performed through a variety of methods (i.e., measurement equations, rule-based techniques, etc.). Given a set of belief-plausibility intervals for each element of the frame of discernment, various methods can be used to select the most likely conclusion. In general, the output at the conclusion of the sensor fusion process need not be restricted to a single state, but could be a combination of states.

Although evidence theory is sometimes referred to as a generalized Bayesian framework, it possesses key differences compared with Bayesian estimation (on which particle filtering is based). Evidence theory allows assignment of probability to an uncertainty class (actually, the union of all propositions), thereby acknowledging uncertainty in the decision process. Bayesian theory does not, requiring that probability mass be divided among singleton propositions even when evidence may point indistinctly to a group of states rather than a single one. Thus, Bayesian theory offers no convenient representation for ignorance or uncertainty and can cause over-committal to individual propositions based on indistinct pieces of evidence. The consideration of nonsingleton sets that evidence theory offers is even more crucial in constructing a flexible estimation framework that avoids large-scale reconfiguration as sensors fluctuate between operational and nonoperational modes, since total belief can be assigned to the uncertainty class whenever a sensor becomes nonoperational. It can be shown that evidence theory collapses to Bayesian theory when unions of propositions are not considered and the frame of discernment is restricted only to singleton propositions.

\section{B. Evidence Theory Sensor Fusion Example}

Consider a very simple case in which two sensors on a flight vehicle measure distance from the ground, and possible values for distance can be either $x_{1}$ or $x_{2}$. Thus, the frame of discernment is given by $\Theta=\left\{\left\{x_{1}\right\},\left\{x_{2}\right\},\left\{x_{1}\right.\right.$ or $\left.\left.x_{2}\right\}\right\}$. The empty set is not included in $\Theta$ because it is assumed that the frame of discernment is exhaustive, meaning $x_{1}$ and $x_{2}$ are the only possible values. Sensor $A$ is used to assign a probability mass function given by

$$
\begin{aligned}
& m_{A}\left(x_{1}\right)=0.7 \quad m_{A}\left(x_{2}\right)=0.1 \\
& m_{A}\left(\left\{x_{1} \vee x_{2}\right\}\right)=0.1 \quad m_{A}(\Theta)=0.1
\end{aligned}
$$

This can intuitively be understood as a $70 \%$ likelihood of $x_{1}$ being the current state, a $10 \%$ likelihood of $x_{2}$ being the current state, a $10 \%$ likelihood that we cannot distinguish between $x_{1}$ and $x_{2}$ but one of them is the current state, and a $10 \%$ chance that the sensor is completely ignorant as to the current state. Sensor $B$ is used to assign a probability mass function given by

$$
\begin{aligned}
& m_{B}\left(x_{1}\right)=0.3 \quad m_{B}\left(x_{2}\right)=0.4 \\
& m_{B}\left(\left\{x_{1} \vee x_{2}\right\}\right)=0.25 \quad m_{B}(\Theta)=0.05
\end{aligned}
$$

Using Eqs. (3) and (4), the belief and plausibility functions can be computed for these two sensors, given by

$$
\begin{array}{ccc}
\operatorname{bel}_{A}\left(x_{1}\right)=0.7 & \operatorname{bel}_{A}\left(x_{2}\right)=0.1 & \operatorname{bel}_{A}\left(\left\{x_{1} \vee x_{2}\right\}\right)=0.9 \\
\operatorname{pl}_{A}\left(x_{1}\right)=0.9 & \operatorname{pl}_{A}\left(x_{2}\right)=0.3 & \operatorname{pl}_{A}\left(\left\{x_{1} \vee x_{2}\right\}\right)=1.0
\end{array}
$$

$$
\begin{array}{lll}
\operatorname{bel}_{B}\left(x_{1}\right)=0.3 & \operatorname{bel}_{B}\left(x_{2}\right)=0.4 & \operatorname{bel}_{B}\left(\left\{x_{1} \vee x_{2}\right\}\right)=0.95 \\
\operatorname{pl}_{B}\left(x_{1}\right)=0.60 & \operatorname{pl}_{B}\left(x_{2}\right)=0.70 & \operatorname{pl}_{B}\left(\left\{x_{1} \vee x_{2}\right\}\right)=1.0
\end{array}
$$

Using Dempster's rule of combination given in Eq. (5), the mass functions of sensors $A$ and $B$ can be combined to form

$$
\begin{aligned}
& m_{A B}\left(x_{1}\right)=0.6957 \quad m_{A B}\left(x_{2}\right)=0.2174 \\
& m_{A B}\left(\left\{x_{1} \vee x_{2}\right\}\right)=0.0797 \quad m_{A B}(\Theta)=0.0072
\end{aligned}
$$

Belief and plausibility functions can then be computed using the fused sensor belief function in (10) and used to determine a state estimate along with a level of confidence associated with that estimate. The manner in which this is performed is called a decision rule. Numerous candidates for decision rules have been proposed by Lee et al. [12]. Ristic and Smets [20], Smets [21,22], and Cobb and Shenoy [23] have further proposed methods of transforming belief functions to probability densities so that decisions can be made in accordance with traditional Bayesian methods.

\section{Conflicting Belief Assignments}

The conflict between belief functions from different sensors is important to characterize in that it represents the disagreement between various sources of information. Conflict determination can help diagnose sensor failures and provide a measure of overall reliability of the resulting estimate. Generally, estimates obtained from combining highly conflicting belief distributions should be viewed as having higher uncertainty. Traditional formulations of belief function theory use the belief assigned to the empty set $\left(k_{\varnothing}\right)$ after application of Dempster's rule as a measure of sensor conflict. For instance, if the belief functions from two sensors have little overlap, application of Dempster's rule results in significant belief $k_{\varnothing}$ being assigned to the empty set (the combined belief function is then normalized using $k_{\phi}$ so that belief assigned to all nonempty set elements adds to 1). However, Jousselme et al. [24] and Martin et al. [25] have shown that nonzero belief is still assigned to the empty set even if Dempster's rule is used to combine two belief functions that are identical (thus, the rule is nonidempotent). Therefore the measure is of limited use since a valid conflict measure would intuitively produce zero if applied to two identical belief distributions.

Numerous authors [24-28] have proposed alternative conflict measures, each with their own unique characteristics. Jousselme et al. [24] and Martin et al. [25] derived a conflict measure based on a distance metric defined for belief functions. Suppose sensors $A$ and $B$ assign belief distributions $m_{A}$ and $m_{B}$ over the same frame of discernment. Let $\mathbf{m}_{A}$ and $\mathbf{m}_{B}$ represent column vectors of these distributions ordered in a given manner. Then the conflict between sensors $A$ and $B$ is defined by a distance metric given by

$$
\operatorname{conf}(A, B)=\sqrt{\frac{1}{2}\left(\mathbf{m}_{A}-\mathbf{m}_{B}\right)^{T} \bar{D}\left(\mathbf{m}_{A}-\mathbf{m}_{B}\right)}
$$

where $\bar{D}$ is a $2^{N} \times 2^{N}$ matrix with elements given by

$$
\bar{D}\left(P_{i}, P_{j}\right)= \begin{cases}1 & \text { if } P_{i}=P_{j}=\varnothing \\ \frac{\left|P_{i} \cap P_{j}\right|}{\left|P_{i} \cup P_{j}\right|} & \text { else }\end{cases}
$$

In Eq. (12), $P_{i}$ and $P_{j}$ represent the proposition combination at element $(\overline{i, j})$ of matrix $\bar{D}$, and the vertical bars represent the cardinality of the set. It can be shown that this conflict measure applied to identical belief distributions will yield 0 , whereas the measure applied to distributions with no focal elements in common will yield 1 .

\section{Evidence Theory State Estimator Design}

This section describes the design of an evidence-theory-based state estimator. The proposed estimator is in some ways a generalization of the particle filter, which considers nonsingleton propositions. First, an overview of the estimator design is presented and key features are highlighted. Then new resampling techniques are described in which the particle set is updated using belief assigned to both singleton and nonsingleton propositions. Conflict measures and their use in fault detection and isolation are described. A final section discusses tractability of the estimation problem.

\section{A. State Estimator Overview}

The primary difference between Bayesian and evidence theories is that evidence theory allows belief to be distributed over both singleton and nonsingleton propositions. Therefore, the estimator presented here has a similar structure to standard implementations of 
the particle filter, with certain key differences that allow handling of nonsingleton sets. A diagram of the estimator is provided in Fig. 1. First, an initial particle set is generated (usually a uniform discretization of the state space). When measurements are obtained, sensors assign belief over the particle set, either to singleton particles or to nonsingleton groups. These belief assignments are then iteratively combined using the conjunctive combination rule. In addition, conflict metrics are determined for each pair of sensors using the distance metric in Eq. (11). Thus, for $n$ sensors, $n(n-1) / 2$ conflict metrics are generated. The singleton state with the highest belief is then chosen as the state estimate at the current time. If the uncertainty class $\Theta$ has the highest belief, the state estimate is set equal to that at the previous time step pending information with less uncertainty. The belief distribution is used in the particle resampling step, which will be described in a subsequent section. Following resampling, particles are propagated to the next measurement time using a nonlinear dynamic model. In addition, conflict measures are used to determine sensor performance, and those producing feedback that is continually in disagreement with the majority of other sensors are removed from the measurement update process.

Note that, like particle filtering, the estimator proposed here discretizes the state space. This is in contrast to Kalman filters, which consider the state space to be continuous and compute estimated values accordingly. Like the particle filter, the evidence-theory-based filter maintains a discrete set of particles representing possible values of the state and thus has somewhat limited resolution for reasonable numbers of particles. However, this discretization of the state space can be beneficial in rejecting bad sensor feedback that lies far outside the bounds of the current particle space. The combined belief mass function computed at each measurement update by the evidencetheory-based filter differs fundamentally from the a posteriori probability density function computed by the particle filter in that the belief mass function cannot be interpreted directly in the context of Bayesian statistics. Numerous authors [21-23] have proposed methods for transforming belief functions to more traditional probability density functions during the decision-making process.

Important differences between the proposed approach and FISSTbased filtering methods developed for multitarget state tracking must also be pointed out. The recursion process for FISST-based filtering methods has the same structure as Bayesian recursion, except integrals of probability densities are replaced by so-called setintegrals, and the densities themselves are replaced by corresponding belief functions [9]. Because of their representation of the multitarget state as a random finite set, the multitarget state and multitarget measurement set within FISST implementations can change in dimensionality over time. In contrast, the filter proposed here does not maintain the same level of generality since it is primarily designed for dynamic system state estimation rather than target tracking. Belief functions are used solely to handle point and set measurements in a rigorous manner and allow for measurement disagreement to be quantified. A set of singleton particles, rather than random finite sets, is used to represent the state posterior density, and the dimension of this particle set is held fixed throughout the filtering process.

A key element of the proposed filter design is sensor fault detection and isolation (FDI) through the use of belief function conflict measures. FDI is not a new idea and has been pursued by numerous researchers exploring fault-tolerant filtering methods. A simple technique for fault rejection in linear systems is an outlier rejection scheme for Kalman filters, in which innovations beyond a specified distance (typically $3 \sigma$ ) from the current state error covariance are not included in measurement updates [29]. Such schemes inherently assume accuracy of filter tuning parameters that may not always be the case and may have trouble distinguishing between measurement outliers and fast dynamic response. Interacting multiple-model (IMM) estimation is another well-known approach [30-32] in which several filters are propagated representing various possible failure modes. Although multiple-model approaches are applicable to both linear [29] and nonlinear systems (through the use of particle filters [33]), computational intensity can be high especially for large numbers of sensors or failure modes. Two strengths of the FDI method proposed here become apparent in comparison with traditional fault-tolerant estimation methods. First, in the proposed method sensor feedback is evaluated independently of filter tuning, and failure probabilities are not required inputs to the sensor-removal process (as they are in many IMM implementations [30]). Second, the computational burden is reduced since conflict measure determination requires less effort than the propagation of multiple filters.

\section{B. Particle Resampling}

Particle resampling must take into account belief assigned to both singleton and nonsingleton propositions. Let $r_{S}$ denote the percentage of combined belief assigned to single particles, and let $N_{T}$ denote the total number of particles. Then $r_{S}$ percent of resampled particles are generated based on the probability density function defined by the single particles and their assigned belief. Note that this is a standard method of resampling for particle filters [34]. Let $N_{S}$ denote the number of particles to be resampled using the singleton density function (i.e., $N_{S}$ is $r_{S}$ percent of $N_{T}$ ). Summarizing this resampling step using singleton propositions at time step $k$, let $x_{k, i}^{+}$ $\left(i=1, \ldots, N_{S}\right)$ denote the particle set after resampling and $x_{k, j}^{-}$ $\left(j=1, \ldots, N_{T}\right)$ the particle set before resampling. Then

$x_{k, i}^{+}=x_{k, j}^{-}$with probability $q_{j} \quad\left(i=1, \ldots, N_{S}\right) \quad\left(j=1, \ldots, N_{T}\right)$

where

$$
q_{j}=\frac{\operatorname{bel}\left(x_{k, j}^{-}\right)}{\sum_{p=1}^{N} \operatorname{bel}\left(x_{k, p}^{-}\right)}
$$

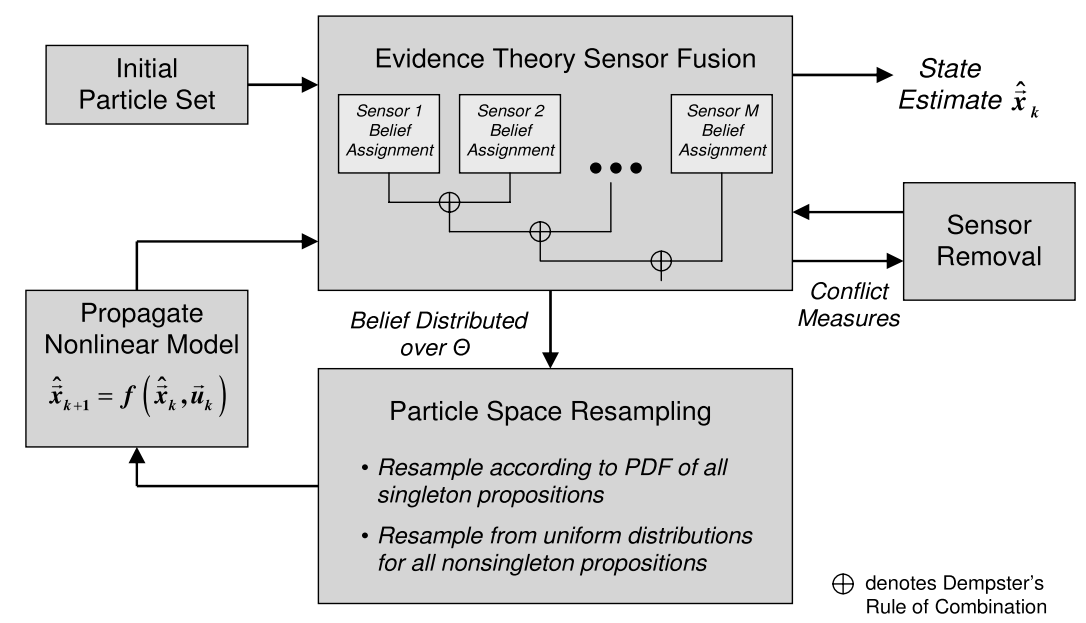

Fig. 1 Diagram of evidence-theory-based state estimator. 
Note that, in general, unless all belief is assigned only to singleton propositions (as is the case for particle filters):

$$
\sum_{p=1}^{N_{T}} \operatorname{bel}\left(x_{k, p}^{-}\right) \neq 1
$$

It can be shown [35] that the probability density of the new particles $x_{k, i}^{+}$tends to the probability density of the previous set of singleton particles as $N_{T} \rightarrow \infty$. The remainder $N_{T}-N_{S}$ particles are generated using belief assigned to nonsingleton propositions. Let $r_{1}, r_{2}, \ldots, r_{n}$ represent the percentages of belief assigned to nonsingleton propositions $p_{1}, p_{2}, \ldots, p_{n}$, respectively. For each nonsingleton proposition $p_{i}, r_{i}$ percent of resampled particles are generated according to a uniform distribution defined by the bounds of the nonsingleton set. Therefore,

$$
x_{k, i}^{+}=s_{i}\left(i=1, \ldots, N_{i}\right)
$$

where $s_{i}$ is a random variable drawn from the uniform distribution $U\left(p_{i}\right)$ and $N_{i}$ represents $r_{i}$ percent of $N_{T}$. To account for the percentage of belief $p_{\Theta}$ assigned to the uncertainty class, $p_{\Theta}$ percent of resampled particles $\left(N_{\Theta}\right)$ are generated using a uniform distribution over the entire state space. When the resampling step is complete,

$$
N_{T}=N_{S}+N_{\Theta}+\sum_{i=1}^{n} N_{i}
$$

total new particles have been generated.

For example, given four particles $\{0.32,0.41,0.45,0.60\}$ for a particular state $\lambda$, suppose belief is distributed according to Table 1 . In this case, 2 particles ( $50 \%$ of the particle set) would be generated from the PDF defined by the singleton particles and their associated belief, one particle ( $25 \%$ of the set) would be generated from a uniform distribution on the interval [0.32, 0.45], and one particle ( $25 \%$ of the set) would be generated from a uniform distribution on the interval $[0.32,0.6]$.

Following particle resampling steps using both singleton and nonsingleton belief distributions, roughening techniques similar to those typically employed in particle filters [34] are used to avoid sample impoverishment. Sample impoverishment occurs when belief assignments from sensors do not overlap well with the given set of particles, and results in the collapse of the particle set to only a few distinct values following resampling. Eventually all particles will collapse to the same value. To avoid this, Gaussian noise is added to the particle set following the resampling process (called roughening). The standard deviation of this noise is termed the roughening coefficient $K_{R}$. Thus, the particle distribution following the roughening step is given by

$$
x_{k, i}^{+}=x_{k, i}^{+}+N\left(0, K_{R}\right)
$$

The roughening coefficient is defined such that it is proportional to the maximum distance between particles at the current time step. This maximum distance $M_{p}$ is given by

$$
M_{p}=\max _{i, j}\left|x_{k, i}^{+}-x_{k, j}^{+}\right|
$$

Table 1 Example particle resampling scenario

\begin{tabular}{cc}
\hline \hline Proposition & Belief \\
\hline Singleton propositions (total 50\% of all belief) \\
$\lambda=0.32$ & 0.12 \\
$\lambda=0.41$ & 0.15 \\
$\lambda=0.45$ & 0.14 \\
$\lambda=0.60$ & 0.09 \\
Nonsingleton propositions (total $50 \%$ of all belief) \\
$\lambda=\{0.32,0.41,0.45\}$ & 0.25 \\
$\lambda=\{0.32,0.41,0.45,0.60\}$ & 0.25 \\
\hline \hline
\end{tabular}

Following standard methods for particle filtering [34], $K_{R}$ is then computed according to

$$
K_{R}=k_{R} M_{p} / N_{T}
$$

where $k_{R}$ is a tuning parameter specifying the amount of noise added to each particle. As a final guard against sample impoverishment, $M_{p}$ is limited such that the value used in (18) cannot drop below a certain threshold. Analogous definitions for higher-dimensional state spaces are provided in [34].

\section{Conflict Measures and Sensor Removal}

At each time step, the conflict measure in Eq. (11) is computed for each pair of $m$ sensors. The result is a symmetric $m \times m$ matrix of conflict measures, which is filtered using a moving average. This filtered set of conflict measures is then analyzed to determine which, if any, sensors are consistently in disagreement with others about the likely current state. This is performed be setting thresholds on acceptable values of the conflict for each sensor pair. If conflict measures for a certain sensor consistently exceed a given threshold, it is eliminated from the measurement update process until conflict measures decrease appropriately. Thus, the sensor diagnostic process assumes that if a sensor is in conflict with the majority of others, the sensor is malfunctioning, or at least incapable or producing reliable information. An analogous procedure could be performed to diagnose problems with an entire group of sensors that may be impaired by computing a mean conflict between sensors of different types.

\section{Tractability of the Estimation Problem}

Given a particle set of size $N$, the total number of elements within the frame of discernment, which includes all singleton propositions and all nonsingleton propositions, is $2^{N}$. When discretizing a state space with reasonable resolution, 100 or more particles are typically used. Thus, the estimator proposed here would theoretically have to consider in excess of $2^{100}$ possible propositions. The estimation problem quickly becomes intractable for any reasonable number of particles. However, given a certain sensor package, only a very small number of nonsingleton propositions will be assigned nonzero belief at any particular time (corresponding to the number of sensors providing belief distributions to nonsingleton set). For instance, the vast majority of sensors will assign belief only to nonsingleton propositions representing a continuous interval of the state space. Thus, for the estimator proposed here, at any given time step the states that must be considered are the particle set $N$ plus a handful of nonsingleton propositions (including the uncertainty proposition). The computational load will therefore be only marginally greater than that required for particle filters [21].

\section{Example Roll Estimator Design}

An example projectile roll-angle estimator is presented. First, sensor and dynamic models are described, with particular emphasis on how belief is distributed over the frame of discernment for each sensor type. Then example estimation results are presented and compared with those from an extended Kalman filter (EKF). A final example explores the case of a malfunctioning sensor, demonstrating the ability of the evidence-theory-based estimator to recognize and remove problematic sensors from the estimation process in real time. Note that a roll estimation example is used here simply to demonstrate how the evidence-theory-based filter can be implemented and to show basic performance characteristics. It is expected that the evidence-theory-based algorithm and the EKF would yield results of similar accuracy for nominal cases, and this is verified.

\section{A. Sensor and Dynamic Models}

The example estimator discussed here is designed for a mortar projectile with a diameter of $81 \mathrm{~mm}$ and weight, axial inertia, and

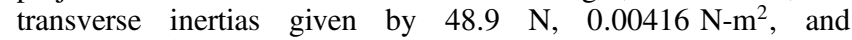
$0.0773 \mathrm{~N}-\mathrm{m}^{2}$, respectively. A projectile body reference frame $B$ is fixed to the projectile such that $\mathbf{I}_{\boldsymbol{B}}$ is aligned with the axis of 


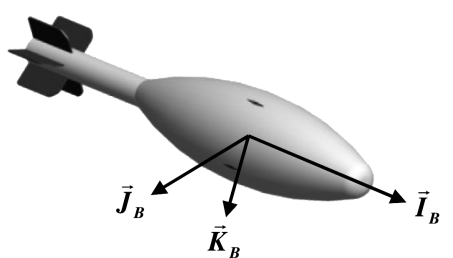

Fig. 2 Projectile body reference frame.

symmetry, and $\mathbf{J}_{\boldsymbol{B}}$ and $\mathbf{K}_{\boldsymbol{B}}$ are directed radially outward, as shown in Fig. 2.

Four types of sensors provide roll feedback to the estimator presented here: magnetometers, thermopiles, GPS, and solar sensors. Each of these types of sensors provides feedback in a unique way with varying levels of resolution, and thus each assigns belief differently over the set of particles representing possible roll states at the current time step. Two sensor types, magnetometers and thermopiles, assign belief primarily to singleton propositions, whereas two other sensors, GPS and solar sensors, assign belief to nonsingleton sets. The projectile is considered to be equipped with two single-axis magnetometers, one thermopile, four solar sensors, and one GPS receiver.

Two single-axis magnetometers are assumed to be located on the body, with their sensitive axes aligned with $\mathbf{J}_{\boldsymbol{B}}$ and $\mathbf{K}_{\boldsymbol{B}}$, respectively. Magnetometer outputs are generated using a simplified model in which the two magnetometer signals are given by

$$
\begin{aligned}
& M_{J}^{*}=S_{y} \tilde{M}_{y}+b_{y}+n_{\mathrm{mag}} \\
& M_{K}^{*}=S_{z} \tilde{M}_{z}+b_{z}+n_{\mathrm{mag}}
\end{aligned}
$$

where $\tilde{M}_{y}$ and $\tilde{M}_{z}$ are components of Earth's magnetic field vector resolved in the $\mathbf{J}_{\boldsymbol{B}}$ and $\mathbf{K}_{\boldsymbol{B}}$ directions, respectively; $S$ is the magnetometer scale factor, $b$ is bias; and $n_{\mathrm{mag}}$ is Gaussian white noise. Since only roll information is desired, outputs $M_{J}^{*}$ and $M_{K}^{*}$ can be reduced to zero-mean sinusoidal signals with unity amplitude, and phase information can be extracted to obtain roll-angle feedback. Thus, at each time step, the signals are first normalized using running estimates of bias and scale factor for each signal obtained from peak detection, yielding normalized outputs $\bar{M}_{J}^{*}$ and $\bar{M}_{K}^{*}$. Harkins [36] has shown that these normalized outputs are the sine and cosine, respectively, of the roll angle offset by a phase angle determined by the magnetic field vector and projectile Euler angles, given by

$\phi_{M}=\tan ^{-1}\left(\frac{M_{N} \sin (\psi)-M_{E} \cos (\psi)}{M_{N} \sin (\theta) \cos (\psi)+M_{E} \sin (\theta) \sin (\psi)+M_{D} \cos (\theta)}\right)$

where $M_{N}, M_{E}$, and $M_{D}$ are the north, east, and down components of the local magnetic field vector. For a given trajectory, a time history of $\phi_{M}$ is generated before flight using trajectory simulation, then uploaded and stored to the guidance system for use in the estimator. Thus, the roll angle can be determined from normalized magnetometer readings according to

$$
\begin{array}{r}
\phi=\sin ^{-1}\left(\bar{M}_{J}^{*}\right)+\phi_{M} \quad \text { or } \quad 180 \mathrm{deg}-\sin ^{-1}\left(\bar{M}_{J}^{*}\right)+\phi_{M} \\
\phi=\cos ^{-1}\left(\bar{M}_{K}^{*}\right)+\phi_{M} \quad \text { or } \quad-\cos ^{-1}\left(\bar{M}_{K}^{*}\right)+\phi_{M}
\end{array}
$$

for the $\mathbf{J}_{\boldsymbol{B}}$ and $\mathbf{K}_{\boldsymbol{B}}$ magnetometers, respectively. Equations (22) and (23) show that for a given magnetometer signal, two roll angles are equally likely.

Magnetometers assign belief to the particle set by assuming sensor noise is Gaussian. Because of the high resolution of this sensor, belief is only assigned to singleton propositions (i.e., single particles). At a given time step, belief is assigned by generating Gaussian distributions (with a standard deviation of $2 \mathrm{deg}$ ) around the two roll angles calculated from Eqs. (22) or (23) such that the total belief adds to 1 . If some belief cannot be assigned due to particle set bounds, the remainder of belief is assigned to the uncertainty class $\Theta$. Figure 3 shows an example belief assignment for a given time step from the $\mathbf{J}_{\boldsymbol{B}}$ magnetometer. Note that the $\mathbf{K}_{\boldsymbol{B}}$ magnetometer produces a similar belief distribution, differing only due to noise and normalization errors.

Thermopiles, like magnetometers, produce a sinusoidally varying signal with roll angle. Rogers et al. [37] have shown that thermopiles measure the thermal gradient between the sky (which appears cold) and the ground (which appears warm). This results in sensor outputs that appear sinusoidal as the projectile rolls with a slightly compressed upper half, as shown in Fig. 4. This compression is more noticeable for pitch angles closer to zero. By normalizing thermopile outputs at each time step, roll-angle estimates can be obtained according to

$$
\phi=\sin ^{-1}\left(\bar{v}^{*}\right)-\alpha \quad \text { or } \quad 180 \operatorname{deg}-\sin ^{-1}\left(\bar{v}^{*}\right)-\alpha
$$

where $\bar{v}^{*}$ represents the normalized thermopile output and $\alpha$ is a phase angle determined by the thermopile offset around the roll axis. Thus, as with magnetometers, thermopiles assign belief only to singleton propositions by creating two Gaussian distributions around the two possible roll angles determined from Eq. (24). In general, one of these peaks will roughly correspond to one of the peaks from the magnetometer distributions, and thus roll angle can be uniquely determined. For the example estimator presented here, one thermopile is considered to be present on the projectile, with the center of its field of view aligned with $\mathbf{J}_{\boldsymbol{B}}$. The thermopile model

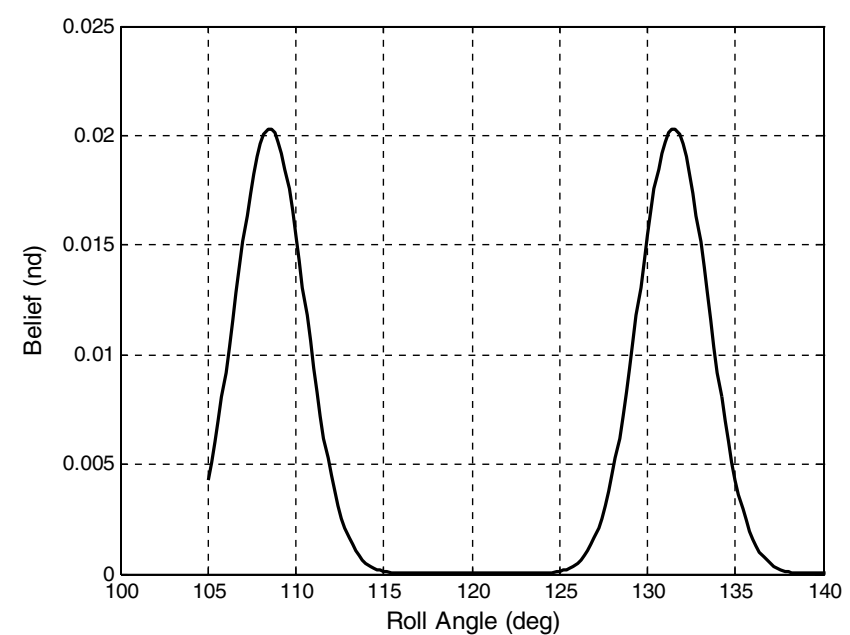

Fig. 3 Example magnetometer belief distribution. Note that the particle set extends from 105 to $140 \mathrm{deg}$, and the remainder of belief is assigned to the uncertainty class.

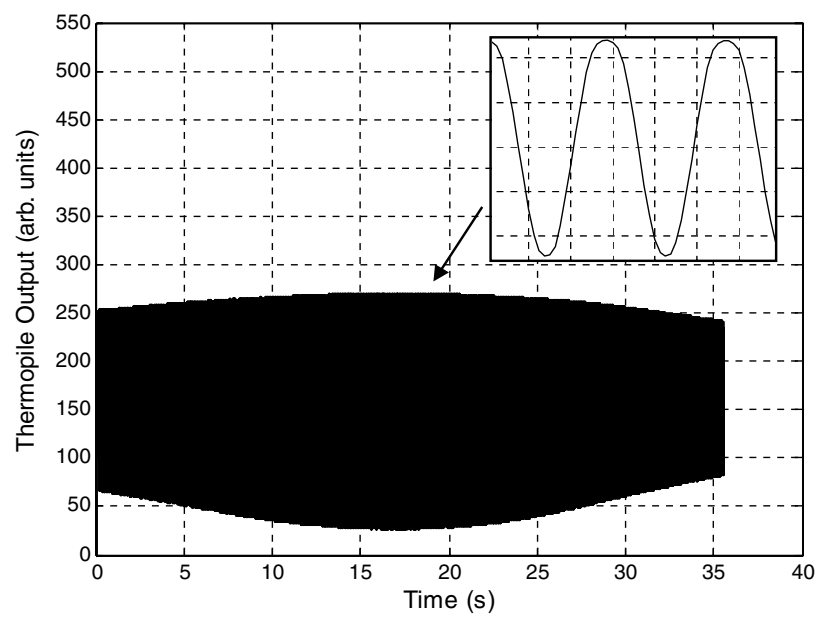

Fig. 4 Example thermopile outputs for $81 \mathrm{~mm}$ mortar projectile trajectory. 
outlined in [37] is used to generate example sensor outputs for the cases shown here. This model has been validated against actual flight data as outlined in the reference.

Roll information for gun-launched projectiles can also be provided through the use of GPS upfinding techniques [38]. Specially equipped GPS receivers can monitor signal strength as the projectile rolls and will provide an output pulse whenever the antenna is pointed within approximately $10 \mathrm{deg}$ of the $\mathbf{K}_{\mathrm{NR}}$ unit vector, where $\mathrm{NR}$ denotes the projectile no-roll reference frame. Note that the NR frame is obtained from the $B$ frame through a transformation of $-\phi$ about the $\mathbf{I}_{B}$ axis. The GPS upfinder is inherently a low resolution sensor, as it provides a window of possible roll angles rather than pointing directly to a single state. Including this information in a precise manner is difficult in a Kalman filter framework, since a continuous measurement equation cannot be defined and outputs simply limit the bounds of the estimate rather than provide direct feedback. It is assumed in this study that the center of the antenna points directly upward along the $-\mathbf{K}_{B}$ axis. Therefore, in the evidence-theory-based state estimator, when a pulse is received the GPS assigns a belief of 1 to the nonsingleton proposition, which includes all roll-angle states between -10 and $10 \mathrm{deg}$, and assigns zero to all other propositions. If no such particles exist in the current set, or if no pulse is present, then a belief of 1 is assigned to the uncertainty class, since the sensor can provide no useful information. Note that assignment of belief to the nonsingleton proposition is not equivalent to assignment of equal belief to all of the component singleton propositions (as is done in a particle filter). Belief in the nonsingleton group of states reflects knowledge that the real state is somewhere within the bounds of the group, but no information is available as to which state is the correct one. Equal assignment to single states implies that the sensor knows that each of the singleton states is equally likely. The use of these two types of assignments in the conjunctive combination rule will produce different results.

Solar sensors are another common method of providing roll orientation [39] information. A pulse is output anytime the vector from the sun to the sensor lies within the narrow field of view of the sensor, as shown in Fig. 5. Note that for a pulse to be generated, the solar vector must intersect the substrate at an angle with magnitude less than $\gamma_{i j}$ in the $\mathbf{I}_{S}-\mathbf{J}_{S}$ plane and with magnitude less than $\gamma_{i k}$ in the $\mathbf{I}_{S}-\mathbf{K}_{S}$ plane. The $S$ frame represents the sensor frame, and $\mathbf{K}_{S}$ is typically aligned with the roll axis of the body, whereas $\mathbf{I}_{S}$ is typically oriented radially outward from the roll axis. The half angle limit $\gamma_{i j}$ is drawn very large in the figure to illustrate sensor operation. In reality, $\gamma_{i j}$ is typically significantly smaller to provide higher roll-angle resolution. The output pulse of the sensor, along with knowledge of the current position of the sun in the sky, allows determination of the roll angle within 1-2 deg when a pulse is obtained. In the studies performed here, values of $\gamma_{i j}=1 \mathrm{deg}$ and $\gamma_{i k}=30 \mathrm{deg}$ are used, and the solar vector is assumed to be completely vertical.

Because they offer an output pulse similar to GPS, solar sensors distribute belief over the particle set by assigning a belief of 1 to the nonsingleton proposition defined by the limits of the possible roll angles given the solar sensor configuration. The width of this nonsingleton interval is normally about $2 \mathrm{deg}$ (given $\gamma_{i j}=1 \mathrm{deg}$ ). If the particle resolution is high, there may be numerous particles

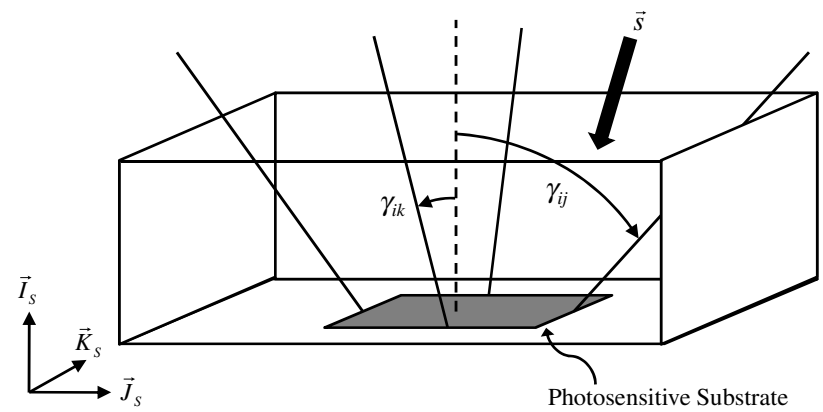

Fig. 5 Solar sensor diagram. Vector s represents the incoming solar vector. within this 2 deg interval. If particle resolution is low, only a single particle may be available within the interval, and belief is thus assigned to this singleton proposition. In the case where no particles are within the interval, or no output pulse is received, a belief of 1 is assigned to the uncertainty proposition and zero to all other propositions.

The sensor belief models described above are used within the estimator to assign belief over the particle set at each measurement update. A dynamic model is also provided to the estimator to propagate the particle set in between updates. For this simple estimator, a linear dynamic model is used in which

$$
\hat{\phi}_{k+1}=\hat{\phi}_{k}+\hat{p} \Delta t
$$

where $\hat{p}$ is the current roll rate and $\Delta t$ is the estimator time step. Note that this model neglects nonlinearities in the kinematic equation for $\phi$, which are typically small for projectile motion. Roll rate $\hat{p}$ is assumed to be provided by a rate gyro aligned with $\mathbf{I}_{\boldsymbol{B}}$. Noise and bias are added to gyro outputs which, along with linearization of the kinematics, introduce error into the dynamic model.

\section{B. Nominal Estimation Results}

Example estimation results are presented for an $81 \mathrm{~mm}$ mortar projectile trajectory. The projectile is launched with a quadrant elevation of $815 \mathrm{mil}$ and a muzzle velocity of $270 \mathrm{~m} / \mathrm{s}$. Altitude vs range and roll-rate time histories are provided in Figs. $\underline{6}$ and 7 , respectively. The sensor suite used for this case are two single-axis magnetometers aligned with the $\mathbf{J}_{\boldsymbol{B}}$ and $\mathbf{K}_{\boldsymbol{B}}$ axes, respectively, a

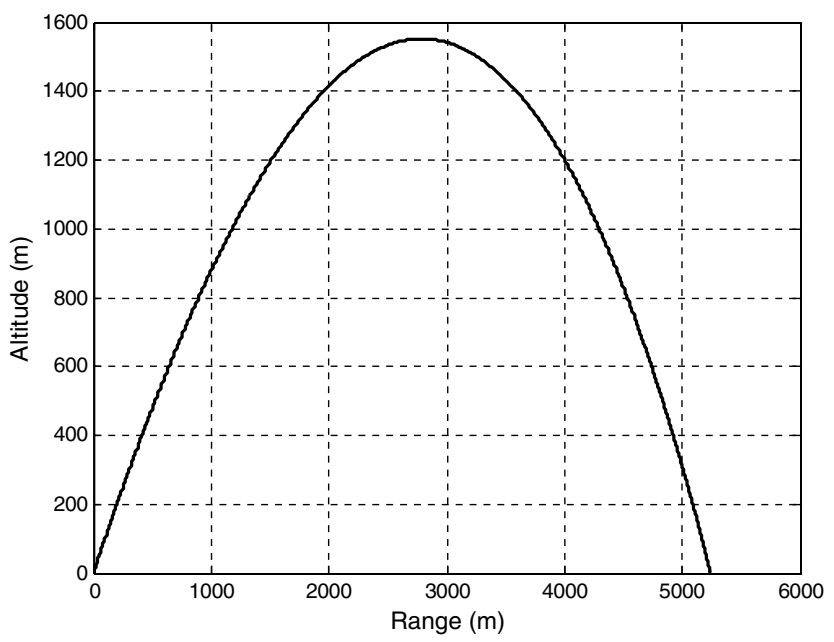

Fig. 6 Range vs altitude for example trajectory.

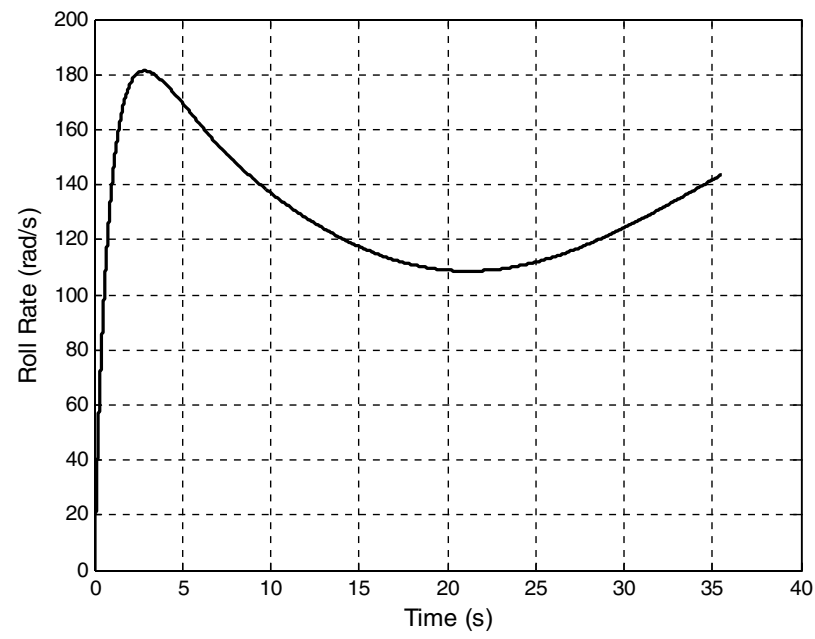

Fig. 7 Roll-rate time history for example trajectory. 
Table 2 Example estimation case error and configuration parameters

\begin{tabular}{lc}
\hline \hline Parameter & Value \\
\hline Gyro noise standard dev & $2 \mathrm{rad} / \mathrm{s}$ \\
Gyro bias & $1 \mathrm{rad} / \mathrm{s}$ \\
Thermopile signal noise standard dev & $1 \mathrm{bit}$ (before normalization) \\
Magnetometer signal noise standard dev & 0.015 normalized units \\
Number of particles & 100 \\
Conflict measure filter order & 50 \\
Filter measurement update rate & $400 \mathrm{~Hz}$ \\
Magnetic field north, east, down components & $\{0.96,0.12,-0.24\}$ \\
Roughening coefficient $k_{R}$ & $20 \mathrm{deg}$ \\
Initial states (evidence theory filter and EKF) & $0 \mathrm{deg}$ \\
EKF initial state error covariance & $1 \mathrm{rad}^{2}$ \\
\hline \hline
\end{tabular}

single thermopile with field of view aligned with $\mathbf{J}_{\boldsymbol{B}}$, four solar sensors with fields of view centered along $\mathbf{J}_{B}, \mathbf{K}_{B},-\mathbf{J}_{B}$, and $-\mathbf{K}_{B}$, respectively, and a GPS upfinding system producing a pulse when $\phi$ is between -10 and $10 \mathrm{deg}$. Table 2 lists error parameters associated with each sensor and other configuration details. An extended Kalman filter was constructed using the sensor and dynamic models described above for performance comparison purposes. GPS upfinding and solar sensor feedback were input into the EKF by selecting a single roll angle to use as the measurement each time a pulse is received (for instance, each time a GPS upfinding pulse is received, a measurement of $\phi=0$ is used).

Figures $\underline{8}$ and $\underline{9}$ show estimation results for the evidence-theorybased estimator and the extended Kalman filter, respectively. Note that in both cases, reasonable estimation performance is verified. The rms error for the EKF case is $1.3 \mathrm{deg}$, whereas rms error for the evidence theory filter is $1.84 \mathrm{deg}$. It is expected that for this filtering problem involving nearly linear dynamics, Kalman filter results will be highly accurate. The evidence theory filter, however, is not an optimal estimator and thus results are not quite as accurate as those generated from the EKF. One factor contributing to estimation error is that Dempster's rule is not robust to sensor belief assignments which, although in general agreement about the approximate location of the state, do not agree closely due to sensor noise or measurement model error. This is analogous to the well-known Zadeh's paradox [40] in which Dempster-Shafer theory provides an incorrect conclusion based on conflicting evidence. Future work will consider the use of more robust extensions of the conjunctive rule to mitigate this problem. Figure 10 shows filtered conflict measures for this nominal case. Note that although relatively constant filtered conflict measures are present throughout the trajectory, higher conflict is present during the middle portion of the trajectory when the thermopile signal has more compressed peaks, thus deviating more from the sinusoidal sensor model and causing more disagreement with magnetometer feedback.

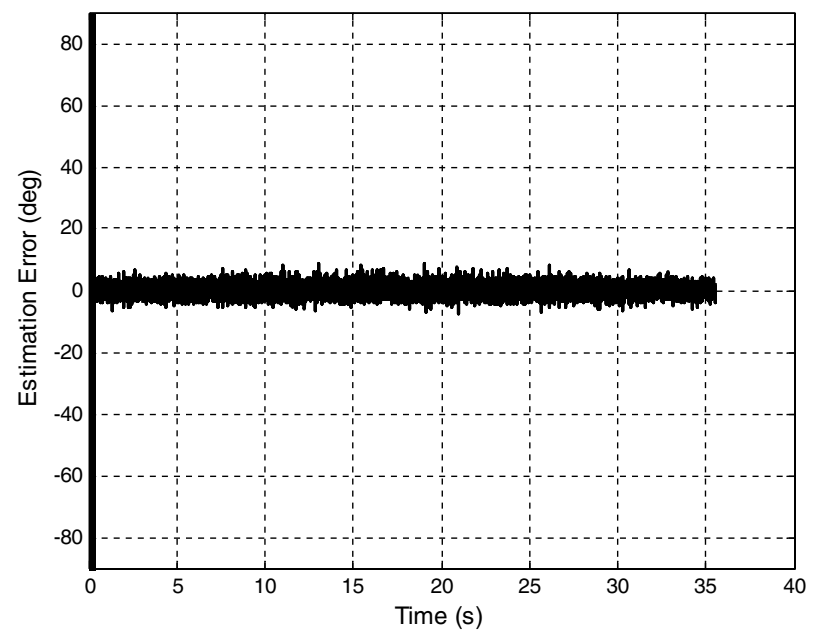

Fig. 8 Roll-angle estimation error, evidence theory filter.

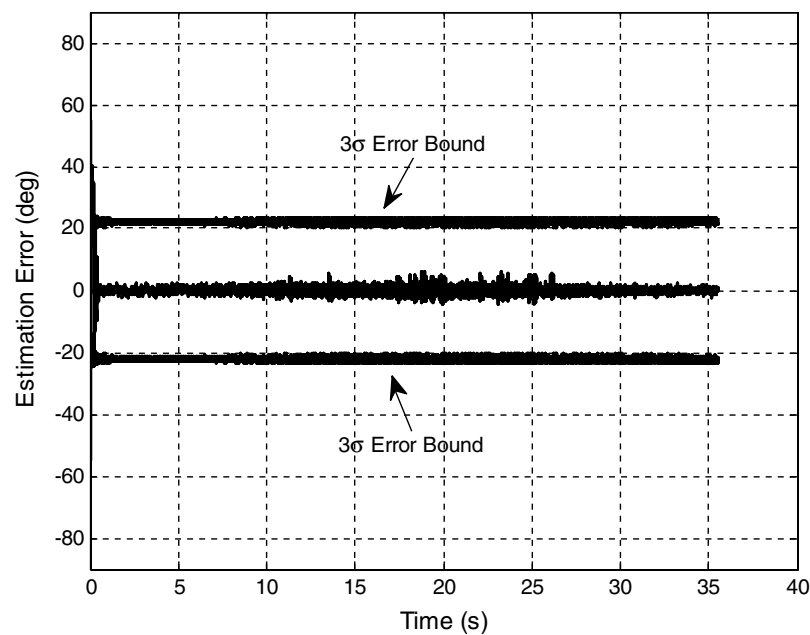

Fig. 9 Roll-angle estimation error, extended Kalman filter.

Like particle filters, the evidence-theory-based filter suffers from significant computational burden in comparison to Kalman filter algorithms, especially as the particle set increases in size. This can be viewed as the price that must be paid for removing the linear dynamic and Gaussian error assumptions inherent in the Kalman filter. For particle filters, it can be shown that larger numbers of particles will generally yield more accurate state estimates. Experiments with the roll estimator developed here actually showed that in cases where sensor errors are high, large particle sets spread out over a sizeable interval of the state space were actually detrimental to filter performance. This is because noisy sensors are able to assign belief to states far from the actual value, instead of being restricted by the particle set bounds and simply assigning belief to the uncertainty proposition. Therefore, the roughening coefficient and particle number must be adjusted appropriately to attempt to "reject" bad feedback from noisy sensors. Future versions of the proposed estimator using more robust combination rules will likely mitigate this issue.

\section{Failed-Sensor Case}

A study demonstrates estimator performance in the presence of a malfunctioning sensor. To highlight the effect of the problematic sensor, the sensor package is reduced to the two magnetometers and the single thermopile in the configuration described in Sec. IV.B. Furthermore, all sensor errors except for the gyro bias and noise are removed. At $20 \mathrm{~s}$ into the trajectory, the magnetometer aligned with $\mathbf{K}_{\boldsymbol{B}}$ malfunctions and produces uniformly random outputs along the interval $[-1,1]$. At $30 \mathrm{~s}$, the sensor regains accuracy. Although this is perhaps not a common failure mode exhibited by magnetometers, it
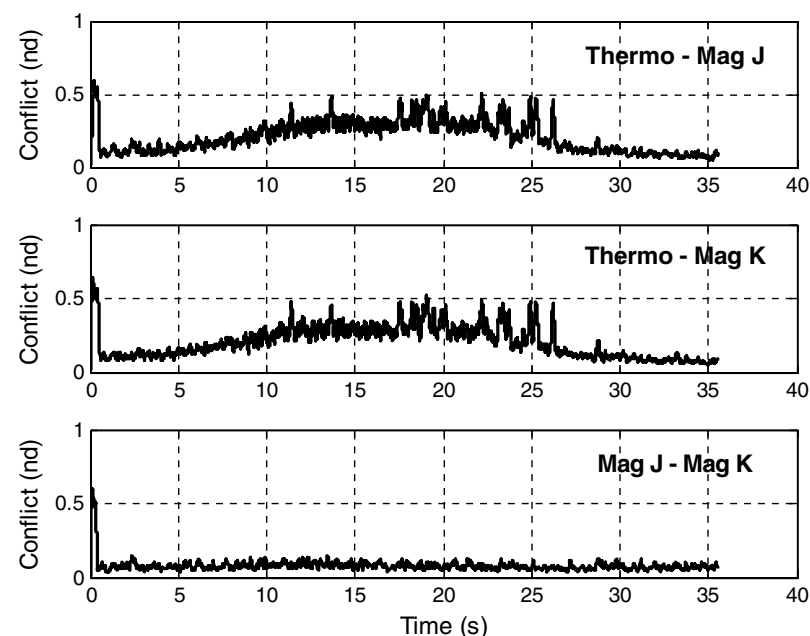

Fig. 10 Filtered conflict measures, evidence theory filter. 
serves to demonstrate how the sensor diagnostic process is performed. Three conflict measures are generated, corresponding to each sensor pair. These conflict measures are monitored, and if more than one exceeds predetermined thresholds, the common sensor to those conflict measures is determined to be malfunctioning and is removed from the measurement update process.

Several cases are simulated to demonstrate the benefit of this sensor diagnostic process. The first is a nominal estimation case in which no sensor failures occur, to be used for comparison purposes. In the second case (termed sensor failure), the sensor fails but is not removed from the estimator. The third case (termed sensor removal) demonstrates performance when conflict measures are monitored and malfunctioning sensors are removed. A fourth case shows estimation results for the malfunctioning sensor case using a standard extended Kalman filter algorithm, also for comparison. A final case shows example results for the failed-sensor case using an EKF that performs outlier rejection, in which measurement innovations outside a $3 \sigma$ deviation from the estimated covariance are excluded from the measurement update [29].

Figure 11 shows the five estimation error time histories. Note that errors are generally very low for all five cases before the sensor malfunction. During the period of sensor malfunction, the sensorfailure case and the standard Kalman filter case continuously exhibit large errors. Note that when the problematic sensor is not removed, the evidence-theory-based estimator shows occasional large error spikes due to the sensitivity of the conjunctive rule to conflicting belief assignments (see discussion in Sec. IV.B). In the evidence theory sensor-removal case, the estimator recognizes high conflict measures and eliminates the $\mathbf{K}_{B}$-axis magnetometer from the update process. This results in significantly reduced error during the period in which the sensor malfunctions, resulting in an overall rms estimation error of $1.52 \mathrm{deg}$. Similar error reduction is demonstrated using the EKF with outlier rejection, although rms errors were higher at $1.62 \mathrm{deg}$. This slight increase in errors is attributed to the fact that the filter still incorporates erroneous measurements if they fall within the $3 \sigma$ boundary and points to the fact that successful outlier rejection inherently requires accurate filter tuning and covariance tracking. Figure 12 shows a time history of all three conflict measures computed for the evidence-theory-based sensor-removal case. Note that a conflict threshold of 0.4 was set to trigger sensor removal. As
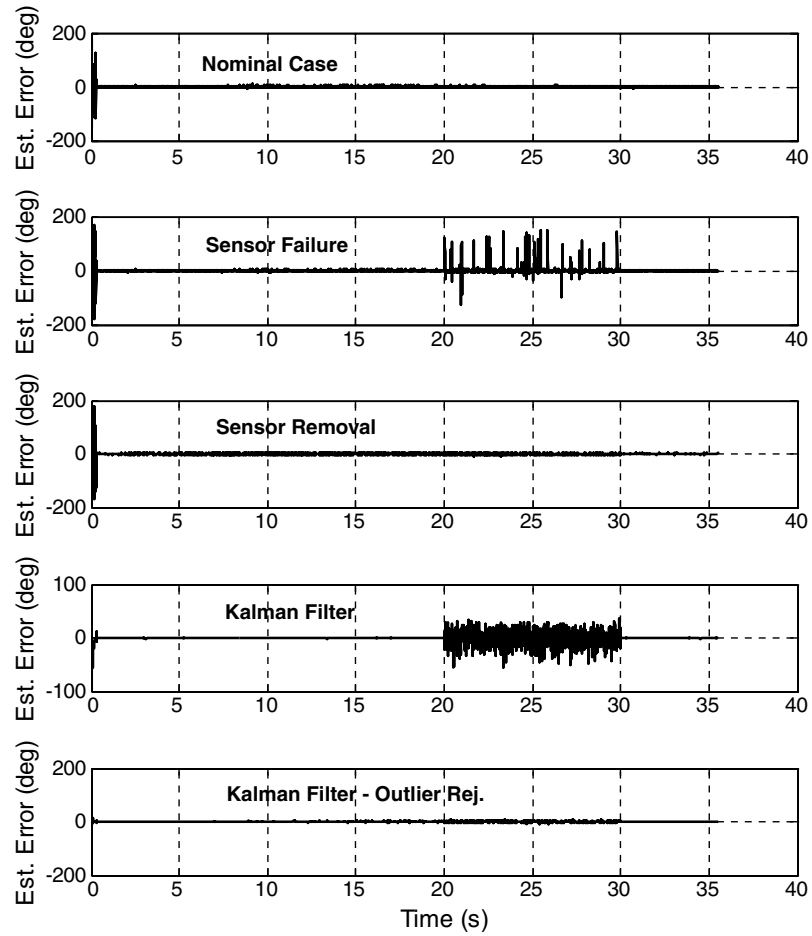

Fig. 11 Estimation error time histories for sensor-malfunction example.
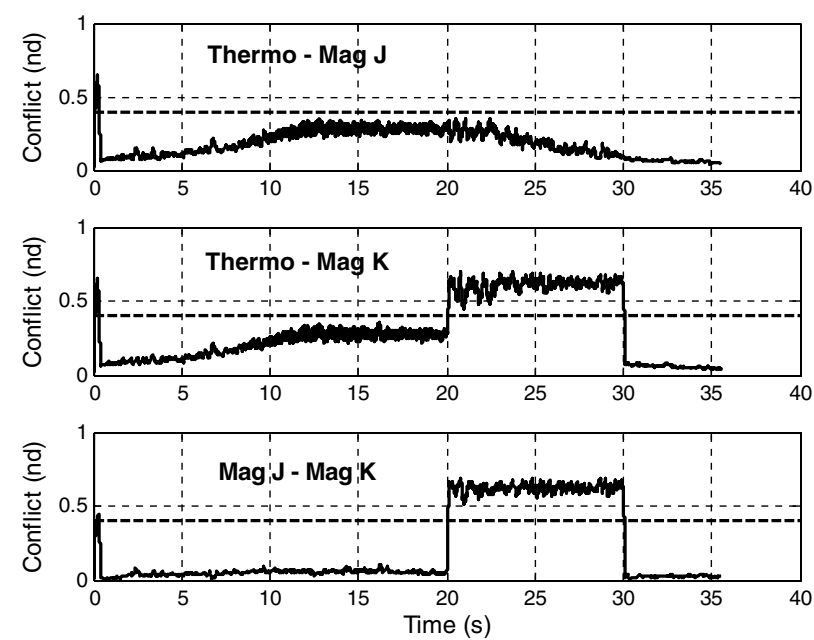

Fig. 12 Filtered sensor conflict metrics for sensor-malfunction example and sensor-removal case. Dashed lines represent thresholds for sensor removal.

shown in Fig. 12, sensor conflict is generally low during periods when all sensors are working. This means that belief assignments from all three sensors are generally close together, which would be expected. When the $\mathbf{K}_{B}$ magnetometer malfunctions, the filtered conflict measures involving the $\mathbf{K}_{B}$ magnetometer quickly rise above the threshold. Noticing that the two high conflict measures both involve the $\mathbf{K}_{B}$ magnetometer, the estimator determines this sensor is malfunctioning and removes it from the update process. After $30 \mathrm{~s}$, the sensor returns online, conflict measures reduce to their normal level, and the sensor is once again included in measurement updates.

This simple example demonstrates how conflict measures can be used to isolate problematic sensors and exclude them from the estimation algorithm. Depending on sensor reliability and the number of sensors involved, this diagnostic process can be a powerful tool that enables significantly increased accuracy. For instance, thermopile disturbances due to weather phenomena typically show up as increased noise in sensor outputs, and this process can be used to mitigate the effect of such disturbances on overall estimation accuracy.

\section{Conclusions}

The design of a novel evidence-theory-based state estimation algorithm has been presented. The use of evidence theory allows processing of all information pertinent to the estimation problem and provides a means to deal with uncertainty in a rigorous way. Unlike in Bayesian estimation, uncertainty in belief assignment is acknowledged and sensors are not forced to distribute belief according to probability densities. At the same time, in contrast to methods derived from Kalman filtering, the estimator can handle fully nonlinear dynamic models and does not make any assumptions regarding sensor error statistics. Furthermore, sensor belief assignments can be contrasted with one another, and problematic sensors can be identified. Removal of these sensors from the estimator requires minimal reconfiguration. The paper begins with an overview of evidence theory, and the proposed estimator design is discussed. Key estimator components, such as resampling, conflict measurements, and sensor belief assignments are described in detail. Results from an example roll estimator demonstrate how the filter is constructed and basic performance characteristics. An example using a faulty magnetometer sensor demonstrates how belief function conflict metrics can be used to systematically identify and remove problematic sensors from the filtering process.

\section{References}

[1] Dempster, A. P., "Upper and Lower Probabilities Induced by a Multiple Valued Mapping," Annals of Mathematical Statistics, AMS-38, 1967, pp. 325-339. 
[2] Dempster, A. P., "A Generalization of Bayesian Inference," Journal of the Royal Statistical Society Series B (Methodological), Vol. 30, No. 2, 1968, pp. 205-247.

[3] Shafer, G., A Mathematical Theory of Evidence, Princeton University Press, Princeton, NJ, 1976.

[4] Dubois, D., and Prade, H., "Possibility Theory as a Basis for Qualitative Decision Theory," Proceedings of the 14th International Joint Conference on Artificial Intelligence, 1995, pp. 1924-1932.

[5] Grabisch, M., and Saveant, P., "Uncertainty Modeling and its Linguistic Expression in Data Fusion Systems," Proceedings of the 1998 IEEE World Congress on Computational Intelligence, 1998, pp. 921-926.

[6] Al-Othman, A. K., "A Fuzzy State Estimator Based on Uncertain Measurements," Measurement, Vol. 42, No. 4, May 2009, pp. 628-637. doi:10.1016/j.measurement.2008.10.007

[7] Layne, J., and Passino, K., "A Fuzzy Dynamic Model Based State Estimator," Fuzzy Sets and Systems, Vol. 122, 2001, pp. 45-72. doi:10.1016/S0165-0114(99)00181-5

[8] Mahler, R., "Nonadditive Probability, Finite Set Statistics, and Information Fusion," Proceedings of the 34th Conference on Decision and Control, New Orleans, LA, Dec. 1995.

[9] Vo, B.-N., Singh, S., and Doucet, A., "Random Finite Sets and Sequential Monte Carlo Methods in Multi-Target Tracking," IEEE Transactions on Aerospace and Electronic Systems, Vol. 10, No. 30, June 2005, pp. 1-19.

[10] Bishop, A., and Jensfelt, P., "Global Robot Localization with Finite Set Statistics," 13th Conference on Information Fusion (FUSION 2010), Edinburgh, Scotland, U.K., July 2010.

[11] Vo, B.-N., Ma, W.-K., "That Gaussian Mixture Probability Hypothesis Density Filter," IEEE Transactions on Signal Processing, Vol. 54, No. 11, Nov. 2006, pp. 4091-4104. doi: $10.1109 /$ TSP.2006.881190

[12] Lee, T., Richards, J., and Swain, P., "Probabilistic and Evidential Approaches for Multisource Data Analysis," IEEE Transactions on Geoscience and Remote Sensing, Vol. GE-25, No. 3, May 1987.

[13] Murphy, R., "Dempster-Shafer Theory for Sensor Fusion in Autonomous Mobile Robots," IEEE Transactions on Robotics and Automation, Vol. 14, No. 2, April 1998, pp. 197-206. doi: $10.1109 / 70.681240$

[14] Yi, Z., Khing, H. Y., Seng, C. C., and Wei, Z. X., "Multi-Ultrasonic Sensor Fusion for Mobile Robots," Proceedings of the IEEE Intelligent Vehicles Symposium, IEEE, Piscataway, NJ, 2000, pp. 387-391.

[15] Wu, H., Siegel, M., Stiefelhagen, R., and Yang, J., "Sensor Fusion Using Dempster-Shafer Theory," IEEE Instrumentation and Measurement Technology Conference, IEEE, Piscataway, NJ, 21-23 May 2002.

[16] Wu, H., Siegel, M., Stiefelhagen, R., and Yang, J., "Sensor Fusion Using Dempster-Shafer Theory II: Static Weighting and Kalman FilterLike Dynamic Weighting," IEEE Instrumentation and Measurement Technology Conference, IEEE, Piscataway, NJ, 20-22 May 2003.

[17] Basir, O., and Yuan, X., "Engine Fault Diagnosis Based on MultiSensor Information Fusion Using Dempster-Shafer Theory," Information Fusion, Vol. 8, 2007, pp. 379-386. doi:10.1016/j.inffus.2005.07.003

[18] Zribi, M., Touil, K., and Benjelloun, M., "Vehicle Localization Via Sensor Fusion Using Evidence Theory," Navigation, Vol. 56, No. 1, 2009, pp. 23-33.

[19] Guan, J. W., and Bell, D. A., Evidence Theory and its Applications, Vol. 1, Studies in Computer Science and Artificial Intelligence, Vol. 7, North Holland, Amsterdam, 1991, p. 82.

[20] Ristic, B., and Smets, P., "The TBM Global Distance Measure for the Association of Uncertain Combat ID Declaration," Information Fusion, Vol. 7, 2006, pp. 276-284 doi:10.1016/j.inffus.2005.04.004

[21] Smets, P., "Data Fusion in the Transferable Belief Model," Proceedings of the Third International Conference on Information Fusion (FUSION 2000), Paris, France, July 2000.

[22] Smets, P., "Decision Making in the TBM: The Necessity of the Pignistic Transformation," International Journal of Approximate Reasoning, Vol. 38, 2005, pp. 133-147. doi:10.1016/j.ijar.2004.05.003

[23] Cobb, B., and Shenoy, P., "On the Plausibility Transformation Method for Translating Belief Function Models to Probability Models," International Journal of Approximate Reasoning, Vol. 41, 2006, pp. 314-380. doi:10.1016/j.ijar.2005.06.008

[24] Jousselme, A. L., Grenier, D., and Bosse, E., "A New Distance Between Two Bodies of Evidence," Information Fusion, Vol. 2, 2001, pp. 91101.

doi:10.1016/S1566-2535(01)00026-4

[25] Martin, A., Jousselme, A. L., and Osswald, C., "Conflict Measure for the Discounting Operation on Belief Functions," 11th International Conference on Information Fusion, Cologne, Germany, JuneJuly 2008

[26] Liu, W., "Analyzing the Degree of Conflict Among Belief Functions," Artificial Intelligence, Vol. 170, 2006, pp. 909-924. doi:10.1016/j.artint.2006.05.002

[27] Tessem, B., "Approximations for Efficient Computation in the Theory of Evidence," Artificial Intelligence, Vol. 61, No. 2, June 1993, pp. 315329. doi:10.1016/0004-3702(93)90072-J

[28] Diaz, J., Rifqi, M., and Bouchon-Meunier, B., "A Similarity Measure Between Basic Belief Assignments," 9th International Conference on Information Fusion, Florence, Italy, 2006.

[29] Biezad, D., Integrated Navigation and Guidance Systems, Vol. 1, AIAA Education Series, AIAA, Reston, VA, pp. 104-105.

[30] Sigalov, D., and Oshman, Y., "A New Formulation of Fault-Tolerant Estimation Problems and Some Solutions," 2010 IEEE Conference of Electrical and Electronics Engineers in Israel, IEEE, Piscataway, NJ, Nov. 2010

[31] De Dona, J. A., Zhuo, X. W., Seron, M., and Olaru, S., "A Fault-Tolerant Multisensor Switching Scheme for State Estimation," 7th IFAC Symposium on Fault Detection, Supervision, and Safety of Technical Processes, Barcelona, Spain, June-July 2009

[32] Lancraft, R. E., and Caglayan, A. K., "A Fault Tolerant Approach to State Estimation and Failure Detection in Nonlinear Systems," Proceedings of the 1982 American Control Conference, IEEE, Piscataway, NJ, 1982, pp. 799-804.

[33] Zhang, Q., Campillo, F., Cerou, F., and LeGland, F., "Nonlinear System Fault Detection and Isolation Based on Bootstrap Particle Filters," Proceedings of the 44th IEEE Conference on Decision and Control and the European Control Conference 2005, IEEE, Piscataway, NJ, 2005

[34] Simon, D., Optimal State Estimation: Kalman, H-Infinity, and Nonlinear Approaches, John Wiley and Sons, Hoboken, NJ, 2006, Chap. 15.

[35] Smith, A., and Gelfand, A., "Bayesian Statistics Without Tears: A Sampling-Resampling Perspective," The American Statistician, Vol. 46, No. 2, May 1992, pp. 84-88. doi: $10.2307 / 2684170$

[36] Harkins, T., "On the Viability of Magnetometer-Based Projectile Orientation Measurements," U.S. Army Research Lab., ARL-TR-4310, Aberdeen Proving Ground, Aberdeen, MD, Nov. 2007.

[37] Rogers, J., Costello, M., and Hepner, D., "Roll Orientation Estimator for Smart Projectiles Using Thermopile Sensors," Journal of Guidance, Control, and Dynamics, Vol. 34, No. 3, May-June 2011, pp. 688-697. doi:10.2514/1.52935

[38] Ilg, M., Fresconi, F., and Maley, J., "GPS Roll Estimation for GunLaunched Spinning Projectiles: Hardware-in-the-Loop Simulation and Experimental Validation," 2011 Joint Navigation Conference, Colorado Springs, CO, June 2011.

[39] Harkins, T., "Understanding Body-Fixed Sensor Output from Projectile Flight Experiments," U.S. Army Research Lab., ARL-TR-3029, Aberdeen Proving Ground, Aberdeen, MD, Sept. 2003.

[40] Benavoli, A., and Antonucci, A., "An Aggregation Framework Based on Coherent Lower Previsions: Application to Zadeh's Paradox and Sensor Networks," International Journal of Approximate Reasoning, Vol. 51, No. 9, Nov. 2010, pp. 1014-1028. doi: $10.1016 /$ j.ijar.2010.08.010

J. Crassidis Associate Editor 